\title{
PERFORMANCE POTENTIAL OF SUGAR BEETS WITH IMPROVED BEETROOT SHAPE IN BREEDING FOR HETEROSIS
}

Parfeniuk O.A., Trush S.H.

Tobacco Experimental Station of the National Research Center "Institute of Agriculture of NAAS", Ukraine

The results of evaluation of the performance of experimental hybrids of sugar beets derived from parents with improved beetroot shape are presented. The sugar beet performance increased with transformation of conical beetroots to oval-conical ones. CMS-based high-yielding hybrids with oval-conical beetroots were created; changes in their biometric parameters were investigated.

Key words: sugar beet, starting material, hybrid, heterosis, beetroot shape, shape index, performance.

Introduction. The development and implementation of breeding innovations aimed to boosting the performance potential of sugar beet plants and their adaptability to environmental conditions in agriculture is one of the main prerequisites of the effective beet growing industry of Ukraine

Increasing energy consumption for growing each additional unit of the crop, limited energy sources, increasing costs for their production require new approaches to the development of resource- and energy-saving technologies in the agrarian sector [1,2].

Creation and introduction in the production of new CMS-based high-yielding hybrids is the most effective way to further increase the productivity of sugar beets. Currently, effective scientific solutions, which will allow for the maximum use of controlled heterosis for the most important economic and valuable features, are needed [3,4]. Therefore, the availability of the genetic diversity of the species Beta vulgaris L., identification of donors of valuable-for- breeding traits and creation, on their basis, of new starting material to generate valuable-for-combinations parents of new high-yielding sugar beet hybrids are especially important challenges in breeding $[5,6]$.

Literature review and problem articulation. Until recently, beet growing was a leading branch of the Ukrainian agribusiness. It largely determined the state of the economy in the agrarian sector and the activities of the domestic sugar market [1,7].

Currently, the Ukrainian sugar beet growing is in a difficult situation. The area under sugar beets has decreased significantly for recent decades. According to the State Statistics Service of Ukraine, in 2020 it amounted to only 218,900 hectares, while in the early 1990 s this crop was sown on more than 1,500,000-1,800,000 hectares in Ukraine [8].

The sugar beet production is sure to have its specifics and complexity, but our country has sufficient potential to regain its position in this area [9, 10]. Consistent intensification of technological processes, increase in beetroot yields and improvement of their quality are the crucial prerequisites for ramping up the sugar beet and sugar production and raising the economic efficiency of the beet industry [2].

The development of breeding and seed production, in particular the creation of CMS-based hybrids combining high yields of beetroots and high sugar content in beetroots, improved technological qualities of raw material for sugar manufacturing, increased resistance to diseases and suitability for intensive technologies, is a most promising way to increase the productivity of sugar beets. 12].

It is known that beetroots of released sugar beet hybrids do not yet fully meet the requirements of the current sugar production. Currently, there are a number of pressing issues related to the beetroot shape that need to be addressed urgently. Excessive embedment into the soil, imperfect shape, and deep grooves significantly increase the energy consumption during harvesting 
and result in the removal of the fertile soil layer from the field. In addition, a significant beetroot mass is lost due to mechanical damage during harvesting $[13,14]$. Hence, the beetroot shape is an important ecological and breeding feature [15].

Accordingly, the creation of sugar beet hybrids with improved beetroot shape will simultaneously increase their productivity and significantly reduce the energy consumption, damage to and soilness of beetroots upon digging out $[14,16]$.

Given the above facts, it is advisable to breed for improved beetroot shape, which opens up prospects for increasing the crop performance and reducing the product costs.

This problem can be solved via introducing fodder beets as donors of valuable-for-breeding and genetic traits in breeding [13]. This makes it possible to expand the genetic potential and improve sugar beets in terms of important economically valuable traits [5].

Fodder beets have a number of genetically determined traits that can be introduced into sugar beets through breeding methods for significant improvement of the latter. Under the same agro-climatic conditions of cultivation, they can give two-fold yields of beetroots in comparison with sugar beets. Almost two thirds of the beetroot are located above the soil surface, which facilitates its digging out and significantly reduces the removal of the fertile soil layer [13]. Therefore, ,sugar beet/fodder beete hybridization for generating new starting material to obtain parents of CMS-based sugar beet hybrids with improved beetroot shape is quite relevant.

Through appropriate selection of crossing components, CMS-based lines and multi-sprout pollinators with altered beetroot shape, a new generation of CMS sugar beet hybrids with higher performance potential can be obtained. Concurrently, the beetroot shape improves, which is also beneficial. Such beetroots are smooth and located partially above the soil surface, having shallow grooves (orthostichy). The beetroot soilness in such hybrids varies $1.9 \%$ to $4.8 \%$, depending on the year of research and soil composition. In addition, the energy consumption for digging them out of the soil is significantly reduced $[16,17]$.

Due to changes in plant genotypes, their varieties are diversified and the performance increases. Therefore, the role of genetic features of modern sugar beet hybrids in the industry intensification is quite significant.

Purpose. To increase the performance of sugar beet hybrids using integrated approaches to the creation, identification and selection of combination-valuable parents; to evaluate the performance potential of experimental hybrids derived from parents with improved beetroot shape.

Materials and methods. The study was conducted in the Laboratory of Sugar Beet Breeding of the Tobacco Experimental Station of the National Research Center "Institute of Agriculture of NAAS" in 2018-2020.

Sixty-six experimental diploid sugar beet hybrids derived from parents of different genetic origin were tested in the field. Crossing was carried out under paired coarse calico bags and in spatially isolated plots.

New starting material of sugar beets was created using classical breeding methods and traditional techniques. The experimental CMS-based sugar beet hybrids were evaluated for several economically valuable traits in accordance with the variety trial method developed by scientists of the Institute of Bioenergy Crops and Sugar Beets NAAS [18]. The record area was $10.8 \mathrm{~m}^{2}$. The experiment was carried out in three replications. The plot arrangement was randomized. Three domestic sugar beet hybrids, Zluka, Bulava and Kvarta, were used as check varieties. Data were statistically processed, as BA Dospekhov described [19].

Results and discussion. The weather in 2018-2020 was instable. Its contrasting nature allowed for comprehensive evaluation of the experimental material, identification of genetic features and phenotypic expression of the most important quantitative traits and selection of forms with highly adaptable plants. During the study period, there were significant deviations of the weather factors from the multi-year averages. In 2018, the amount of precipitation was similar to the multi-year average $(633 \mathrm{~mm})$, while 2019 and 2020 were quite arid (380 and $483 \mathrm{~mm}$, respectively). In the spring and summer, the precipitation amounts were lower than the multi-year average: $29-47 \%$ and $24-53 \%$ related to the average, respectively. The 2018 and 2020 autumns were quite wet (exceeding the multi-year average by $56.8 \%$ and $44.7 \%$, respectively), and the 2019 autumn was (44.3\% of the multi-year average). The air temperature in 2018-2020 significantly 
exceeded the multi-year average (by $7.4^{\circ} \mathrm{C}$ ). The vegetation periods of plants in the study years were slightly arid $(\mathrm{HTC}=0.66-0.95)$. In general, they were favorable for the normal growth and development of sugar beet plants.

To evaluate the effectiveness of the developed trends and designs of the breeding of sugar beet initial forms (O-type lines and their CMS analogues, diploid multi-sprout pollinators) with improved beetroot shape, we created experimental CMS-based hybrids, where female components were pre-selected high-sugar CMS-lines of genetic origin with conical beetroots and new CMSlines with improved beetroot shape (oval-conical), and male components were $\mathrm{BC}_{1}$ multi-sprout pollinators with oval-conical beetroots and initial accessions of diploid multi-sprout pollinators with conical and wide-conical beetroots.

Analysis of the performance of sugar beet hybrids originating from the parents with improved beetroot shape (oval-conical) shows that the beetroot yields, collection and sugar output from the hybrids are significantly better than the group check accession (Table 1).

Table 1

Performance of the best experimental sugar beet hybrids originating from CMS lines and multi-sprout pollinators $\left(\mathrm{BC}_{1}\right)$ with oval-conical beetroots, 2018-2020.

\begin{tabular}{|c|c|c|c|c|c|c|c|c|c|}
\hline \multirow[b]{2}{*}{$\begin{array}{l}\text { Breeding } \\
\text { accession }\end{array}$} & \multirow[b]{2}{*}{ Cross combination } & \multirow[b]{2}{*}{$\begin{array}{l}\text { Yield, } \\
\text { t/ha }\end{array}$} & \multirow{2}{*}{$\begin{array}{c}\text { Sugar } \\
\text { content, } \\
\%\end{array}$} & \multirow{2}{*}{$\begin{array}{c}\text { Sugar } \\
\text { collec- } \\
\text { tion, } \mathrm{t} / \mathrm{ha}\end{array}$} & \multirow{2}{*}{$\begin{array}{c}\text { Sugar } \\
\text { output, } \\
\text { t/ha }\end{array}$} & \multicolumn{4}{|c|}{$\begin{array}{c}\text { Related to the group check accession, } \\
\%\end{array}$} \\
\hline & & & & & & Yield & $\begin{array}{c}\text { Sugar } \\
\text { content }\end{array}$ & $\begin{array}{c}\text { Sugar } \\
\text { collectio } \\
\mathrm{n} \\
\end{array}$ & $\begin{array}{l}\text { Sugar } \\
\text { output }\end{array}$ \\
\hline E72171 & $\begin{array}{l}\text { Um.TsChS4/130.1P } \\
\text { x BZ(1729/21x1705) } \\
/ b_{1} \mathrm{P}\end{array}$ & 58.4 & 17.6 & 10.28 & 8.77 & 120.1 & 97.2 & 116.8 & 116.5 \\
\hline E72172 & $\begin{array}{l}\text { Um.TsChS32/130.1P } \\
\text { x BZ 33/ } \mathrm{b}_{1} \mathrm{P}\end{array}$ & 56.6 & 18.0 & 10.19 & 8.67 & 116.5 & 99.4 & 115.8 & 115.0 \\
\hline E72176 & $\begin{array}{l}\text { Um. ChS37/130.1P x } \\
\text { BZ 76/ b } 1 \text { P }\end{array}$ & 59.6 & 17.7 & 10.55 & 9.05 & 122.6 & 97.8 & 119.9 & 120.2 \\
\hline E72177 & $\begin{array}{l}\text { Um.ChS42/130.2P x } \\
\mathrm{BZ}(1729 / 21 \times 1705) \\
\mathrm{b}_{1} \mathrm{P}\end{array}$ & 58.4 & 17.8 & 10.40 & 8.89 & 120.1 & 98.3 & 118.2 & 118.1 \\
\hline E72180 & 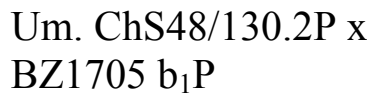 & 59.8 & 17.8 & 10.64 & 9.19 & 123.0 & 98.3 & 120.9 & 122.0 \\
\hline E72181 & $\begin{array}{l}\text { Um. ChS51/130.2P x } \\
\text { BZ51997 b } 1 \text { P }\end{array}$ & 58.0 & 17.7 & 10.27 & 8.86 & 119.3 & 97.8 & 116.7 & 117.7 \\
\hline E72186 & $\begin{array}{l}\text { Um. ChS54/130.2P x } \\
\text { BZ76 } \mathrm{b}_{1} \mathrm{P}\end{array}$ & 57.4 & 17.9 & 10.27 & 8.82 & 118.1 & 98.9 & 116.7 & 117.1 \\
\hline E72191 & $\begin{array}{l}\text { Um. ChS67/141P x } \\
\mathrm{BZ}(1729 / 21 \times 1705) \\
\mathrm{b}_{1} \mathrm{P}\end{array}$ & 56.6 & 17.9 & 10.13 & 8.68 & 116.5 & 98.9 & 115.1 & 115.3 \\
\hline E72193 & $\begin{array}{l}\text { Um. ChS69/141P x } \\
\text { BZ51997 b } \mathrm{P}\end{array}$ & 60.0 & 17.5 & 10.50 & 9.07 & 123.3 & 96.7 & 119.3 & 120.4 \\
\hline E72126 & $\begin{array}{l}\text { Um. ChS76/141P x } \\
\text { BZ } 33\end{array}$ & 59.7 & 17.6 & 10.51 & 8.98 & 121.8 & 97.2 & 119.4 & 119.3 \\
\hline E72132 & $\begin{array}{l}\text { Um. ChS79/141P x } \\
\text { BZ } 33 \mathrm{~b}_{1} \mathrm{P}\end{array}$ & 59.9 & 17.8 & 10.66 & 9.07 & 122.8 & 98.3 & 121.1 & 120.4 \\
\hline E72135 & $\begin{array}{l}\text { Um. ChS83/141P x } \\
\text { BZ } 76 \mathrm{~b}_{1} \mathrm{P}\end{array}$ & 57.9 & 17.5 & 10.13 & 8.83 & 119.4 & 96.7 & 115.1 & 117.2 \\
\hline E72136 & $\begin{array}{l}\text { Um.ChS89/84P x } \\
\text { BZ } 51997 b_{1} P\end{array}$ & 59.1 & 17.5 & 10.34 & 9.04 & 121.6 & 97.2 & 117.5 & 120.0 \\
\hline E72151 & $\begin{array}{l}\text { Um.ChS96/84P x } \\
\text { BZ } 33 \mathrm{~b}_{1} \mathrm{P}\end{array}$ & 60.0 & 17.6 & 10.56 & 9.05 & 123.4 & 97.8 & 120.0 & 120.2 \\
\hline$\overline{\bar{x}}$ & & 58.7 & 17.7 & 10.39 & 8.93 & 120.6 & 97.9 & 118.0 & 118.5 \\
\hline Group ch & leck accession & 48.6 & 18.1 & 8.50 & 7.53 & - & - & - & - \\
\hline$L S D_{05}$ & & 3.51 & 0.49 & 0.52 & 0.54 & - & - & - & - \\
\hline
\end{tabular}


The sugar content in their beetroots was lower than or similar to the group check accession. In general, the yielding type (E) of performance is intrinsic to both parents and their hybrids. As to beetroot yield, they exceeded the group check accession by $16.5-23.4 \%$; the sugar collection was higher by $15.1-21.1 \%$; and the sugar output - by $15.0-22.0 \%$.

To date, parentse combining ability for the performance traits was the main criterion for the selection of sugar beet CMS-based hybridse parents. Combining ability is a hereditary trait, allowing for selection for high combinatorial value as well as for other quantitative traits. Given this factor and the fact that combination-capable multi-sprout pollinators (BZ) were the female components of $F_{1}$ sugar-feed hybrids, we can assert that the initial accessions, i.e. multi-sprout pollinators $(\mathrm{BZ})$, and their offspring $\left(\mathrm{BC}_{1}\right)$ with improved beetroot shape did not differ significantly in terms of the genetic control and expression of this trait. Here, modified beetroot shape was the main factor in increasing the performance of the experimental CMS-based sugar beet hybrids. in Table 2.

The results of studying the beetroot shape in best experimental sugar beet hybrids are shown

Table 2

Beetroot shape in the best experimental sugar beet hybrids, 2018-2020

\begin{tabular}{|c|c|c|c|c|c|c|c|c|c|}
\hline $\begin{array}{c}\text { Bree- } \\
\text { ding } \\
\text { acces- } \\
\text { sion }\end{array}$ & Cross combination & $\mathrm{L}, \mathrm{cm}$ & $\mathrm{D}, \mathrm{cm}$ & $\mathrm{d}, \mathrm{cm}$ & $\mathrm{B}, \mathrm{cm}$ & K & $\begin{array}{l}\text { Beetroot } \\
\text { shape } \\
\text { index (S) }\end{array}$ & $\begin{array}{l}\text { Beetroot } \\
\text { shape }\end{array}$ & $\begin{array}{l}\text { Embedment } \\
\text { into the soil }\end{array}$ \\
\hline E72171 & $\begin{array}{l}\text { Um.TsChS4/130.1P x } \\
\mathrm{BZ}(1729 / 21 \mathrm{x} \\
1705) / \mathrm{b}_{1} \mathrm{P}\end{array}$ & 21.2 & 9.5 & 1.0 & 4.3 & 0.61 & 1.18 & $\begin{array}{l}\text { Oval- } \\
\text { conical }\end{array}$ & $3 / 4$ \\
\hline E72172 & $\begin{array}{l}\text { Um.TsChS32/130.1P } \\
\text { x BZ 33/ b } 1 \text { P }\end{array}$ & 20.8 & 9.2 & 1.0 & 4.1 & 0.63 & 1.14 & $\begin{array}{l}\text { Oval- } \\
\text { conical }\end{array}$ & $3 / 4$ \\
\hline E72176 & $\begin{array}{l}\text { Um. ChS37/130.1P x } \\
\text { BZ } 76 / b_{1} P\end{array}$ & 23.3 & 9.2 & 1.0 & 4.3 & 0.66 & 1.12 & $\begin{array}{l}\text { Oval- } \\
\text { conical }\end{array}$ & $3 / 4$ \\
\hline E72177 & $\begin{array}{l}\text { Um.ChS42/130.2P x } \\
\mathrm{BZ}(1729 / 21 \mathrm{x} 1705) \\
\mathrm{b}_{1} \mathrm{P}\end{array}$ & 20.7 & 9.0 & 1.0 & 4.1 & 0.65 & 1.16 & $\begin{array}{l}\text { Oval- } \\
\text { conical }\end{array}$ & $3 / 4$ \\
\hline E72180 & $\begin{array}{l}\text { Um. ChS } 48 / 130.2 P \times \\
\text { BZ1705 b }\end{array}$ & 22.3 & 9.5 & 1.0 & 4.3 & 0.66 & 1.21 & $\begin{array}{l}\text { Oval- } \\
\text { conical }\end{array}$ & $3 / 4$ \\
\hline E72181 & $\begin{array}{l}\text { Um. ChS51/130.2P x } \\
\text { BZ51997 b } 1 \text { P }\end{array}$ & 22.2 & 9.4 & 1.0 & 4.3 & 0.64 & 1.17 & $\begin{array}{l}\text { Oval- } \\
\text { conical }\end{array}$ & $3 / 4$ \\
\hline E72186 & $\begin{array}{l}\text { Um. ChS54/130.2P x } \\
\text { BZ76 b } 1 \text { P }\end{array}$ & 21.7 & 9.1 & 1.0 & 4.2 & 0.64 & 1.13 & $\begin{array}{l}\text { Oval- } \\
\text { conical }\end{array}$ & $3 / 4$ \\
\hline E72191 & $\begin{array}{l}\text { Um. ChS67/141P x } \\
\text { BZ(1729/21x1705) } \\
b_{1} \mathrm{P}\end{array}$ & 20.8 & 9.4 & 1.0 & 3.9 & 0.63 & 1.11 & $\begin{array}{l}\text { Oval- } \\
\text { conical }\end{array}$ & $3 / 4$ \\
\hline E72193 & $\begin{array}{l}\text { Um. ChS69/141P x } \\
\text { BZ51997 b } \mathrm{P}\end{array}$ & 22.6 & 9.3 & 1.0 & 4.2 & 0.68 & 1.18 & $\begin{array}{l}\text { Oval- } \\
\text { conical }\end{array}$ & $3 / 4$ \\
\hline E72126 & $\begin{array}{l}\text { Um. ChS76/141P x } \\
\text { BZ } 33\end{array}$ & 22.9 & 9.4 & 1.0 & 4.2 & 0.66 & 1.14 & $\begin{array}{l}\text { Oval- } \\
\text { conical }\end{array}$ & $3 / 4$ \\
\hline E72132 & $\begin{array}{l}\text { Um. ChS79/141P x } \\
\text { BZ } 33 \mathrm{~b}_{1} \mathrm{P}\end{array}$ & 23.1 & 9.5 & 1.0 & 4.5 & 0.67 & 1.24 & $\begin{array}{l}\text { Oval- } \\
\text { conical }\end{array}$ & $3 / 4$ \\
\hline E72135 & $\begin{array}{l}\text { Um. ChS83/141P x } \\
\text { BZ } 76 \mathrm{~b}_{1} \mathrm{P}\end{array}$ & 21.4 & 9.4 & 1.0 & 4 & 0.64 & 1.12 & $\begin{array}{l}\text { Oval- } \\
\text { conical }\end{array}$ & $3 / 4$ \\
\hline E72136 & $\begin{array}{l}\text { Um.ChS89/84P x } \\
\text { BZ } 51997 b_{1} P\end{array}$ & 22.7 & 9.5 & 1.0 & 4.4 & 0.66 & 1.22 & $\begin{array}{l}\text { Oval- } \\
\text { conical }\end{array}$ & $3 / 4$ \\
\hline E72151 & $\begin{array}{l}\text { Um.ChS96/84P x } \\
\text { BZ } 33 \mathrm{~b}_{1} \mathrm{P}\end{array}$ & 22.1 & 9.3 & 1.0 & 4.1 & 0.65 & 1.12 & $\begin{array}{l}\text { Oval- } \\
\text { conical }\end{array}$ & $3 / 4$ \\
\hline & $\overline{\boldsymbol{x}}$ & 22,0 & 9.3 & 1.0 & 4.2 & 0.65 & 1.16 & - & - \\
\hline \multirow{2}{*}{\multicolumn{2}{|c|}{$\begin{array}{l}\text { Group check accession } \\
L S D_{05}\end{array}$}} & 20,1 & 9.1 & 1.0 & 2.6 & 0.52 & 0.61 & - & Embedded \\
\hline & & 1,10 & 0.47 & - & 0.21 & 0.03 & 0.06 & - & - \\
\hline
\end{tabular}


Notes: L - beetroot length; $\mathrm{D}$ - beetroot maximum diameter; $\mathrm{d}$ - beetroot tail diameter; In distance from the maximum diameter plane to the beetroot top, where the bud begins to form; $\mathrm{K}$ beetroot weight coefficient.

Analysis of the beetroot shape in such hybrids shows that they were all oval-conical. The beetroot shape index varied 1.11 to 1.24 . Beetroots of these hybrids were embedded into the soil by $3 / 4$ of their length, had a smooth surface and small grooves (orthostichy). The sugar beet hybrids used as check accessions had conical beetroots, fully embedded into the soil. In the check accessions, the average shape index $\mathrm{S}$ was 0.61 .

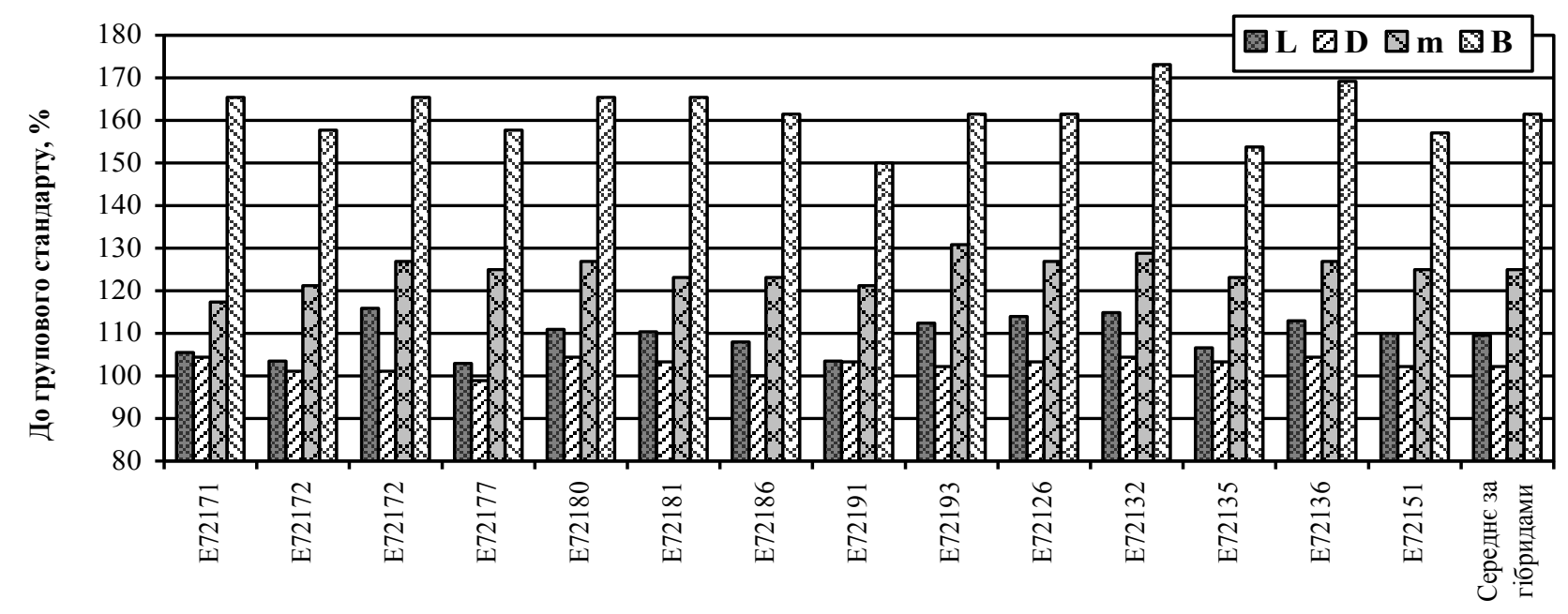

Fig. 1. Modified beetroot shape in the experimental hybrids compared to the group check accession

In the sugar beet hybrids derived from the parents with improved root shape (oval-conical), beetroots were longer (L) by $9.5 \%$ and larger in the diameter (D) by $2.2 \%$; the distance from the maximum diameter plane to the beetroot top (B) increased by $61.5 \%$ compared to the group check accession. In parallel with changes of the beetroot shape, the beetroot weight $(\mathrm{m})$ increased by $25.0 \%$. These facts indicate that it is possible to increase the performance potential of sugar beet CMS-based hybrids due to widespread involvement in breeding of fodder beets as donors of valuable-for-breeding and genetic traits.

In general, all this confirms our hypothesis that the transition from conical beetroots to ovalconical ones to an increase in the performance of the sugar beet hybrids by $15-21 \%$.

Using the study results, were developed competitive sugar beet CMS-based hybrids with oval-conical beetroots, which partially protrude above the soil surface and have a smooth surface and shallow grooves (orthostichy).

Conclusions. We have created 14 high-yielding sugar beet CMS-based hybrids with improved beetroot shape. It was established that the transition from conical beetroots to ovalconical ones in parents resulted in increased performance of sugar beet hybrids. The beetroot yields from the hybrids were by $16.5-23.4 \%$ higher than from the group check accession, the sugar collection was by $15.1-21.1 \%$ higher, and the sugar output - by $15.0-22.0 \%$ higher.

\section{Список використаних джерел}

1. Зубенко В.Ф., Роїк М.В., Іващенко О.О., Гізбулін Н.Г. та ін. Буряківництво. Проблеми інтенсифікації та ресурсозбереження. За ред. В.Ф. Зубенка. Київ: НВП ТОВ "Альфастевія ЛТД", 2007. 486 с. 
2. Томашевська О.А., Петриняк Н. С. Проблеми розвитку та підвищення економічної ефективності виробництва цукрових буряків. Проблеми економіки. 2017. № 2. С. 347352.

3. Роїк М.В., Корнєєва М.О. Напрями, методи та стратегія розвитку селекції цукрових буряків. Цукрові буряки. 2015. № 6. С. 7-9.

4. Корниенко А.В., Моргун А.В., Труш С Г., Селекция свеклы на гетерозис (Beta vulgaris L.). Воронеж, 2007. 255 c.

5. Роїк М.В., Корнєєва М.О., Ермантраут Е.Р. Формування елементів продуктивності у цукрових буряків залежно від типу генних взаємодій. Вісник аграрної науки. 1997. № 9. C.53-56.

6. Frese L., Desprez B., Ziegler D. Potential of genetic resources and breeding strategies for basebroadening in Beta. Broadening the genetic base of crop production/ eds. Cooper H.D., Spillane C., Hodgkin T. UK: Wallingford, 2001. 295-309 p. DOI: 10.1079/9780851994116.0295.

7. Глеваський І.В. Буряківництво: Навч.посібник. Київ: Вища школа, 1991. 620 с.

8. Державна служба статистики України. URL: http://www.ukrstat.gov.ua.

9. Саблук П.Т., Коденська М.Ю., Власов В.І. та ін. Цукробурякове виробництво України: проблеми відродження, перспективи розвитку: монографія /за ред. акад. П.Т. Саблука, проф. М.Ю. Коденської. Київ: ННЦ ІАЕ, 2007. 390 с.

10. Данилишин М.С. Ефективність діяльності бурякоцукрової галузі України. Економіка та держава. 2016. № 1. С. 108-111.

11. Роїк М.В., Корнєєва М.О. Гібриди нового покоління цукрового буряку і їхня роль у процесі інтенсифікації галузі. Сортовивчення та охорона прав на сорти рослин. 2006. № 3. C. 71-82. DOI: 10.21498/2518-1017.3.2006.67681.

12. Чекалін M.М., Тищенко В.М., Баташова М.Є. Селекція та генетика окремих культур. Полтава, 2008. 368 с.

13. Перетятько В.Г., Боршківський I.М. Селекція на удосконалення форми і розмірів коренеплодів. Цукрові буряки. 2002. № 3. С. 16-21.

14. Роїк М.В., Зуєв М.М., Борисюк В.О. Про морфологічні ознаки рослин цукрових буряків та їх вплив на продуктивність і якість механізованого збирання. Збірник наукових праџь Інституту иукрових буряків УААН. 2000. Вип.2(2). С. 146-166.

15. Роїк М.В., Корнєєва М.О. Форма коренеплодів як важлива еколого-селекційна ознака цукрових буряків. Підвищення ефективності бурякового виробництва та проблеми екології і відходів. Київ, 1994. С. 33-34.

16. Tsialtas J.T., Maslaris N. Sugar beet root shape and its relation with yield and quality. Sugar Tech. 2010. No 12(1). P. 47-52.

17. Хоменко O.I, Пушанко М.M. Взаємозалежність геометричних характеристик та зв"язаної забрудненості коренеплодів цукрових буряків. Харчова промисловість. 2008. № 6. С. $12-$ 15.

18. Роїк М.В., Гізбуллін Н.Г., Сінченко В.М., Присяжнюк О.І. та ін. Методики проведення досліджень у буряківництві. За. ред. М.В. Роїка та Н.Г. Гізбулліна. Київ : ФОП Корзун Д.Ю., 2014. 374 c.

19. Доспехов Б.А. Методика полевого опыта с основами статистической обработки результатов исследований. Москва: Агропромиздат, 1985. 351 с.

\section{References}

1. Zubenko VF, Royik MV, Ivashchenko OO, Gizbulin NG. Beet growing. Problems of intensification and resource saving. Zubenko VF, ed. Kyiv: Alpha-stevia LTD; 2007. 486 p.

2. Tomashevska OA, Petrynyak NS. Problems of development and increase of the economic efficiency of sugar beet production. Problemy ekonomiky. 2017; 2: 347-352.

3. Royik MV, Korneeva MO. Trends, methods and strategy of sugar beet breeding development. Tsukrovi buryaky. 2015; 6: 7-9.

4. Korniyenko AV, Morhun AV, Trush SH. Beet breeding for heterosis (Beta vulgaris L.). Voronezh, 2007. 255 p.

5. Royik MV, Korneeva MO, Ermantraut ER. Performance constituents in sugar beets depending 
on the type of gene interactions. Visnyk agrarnoi nauky. 1997; 9: 53-56.

6. Frese L, Desprez B, Ziegler D. Potential of genetic resources and breeding strategies for basebroadening in Beta. In: Cooper HD, Spillane C, Hodgkin T, editors. Broadening the genetic base of crop production. UK: Wallingford, 2001. P. 295-309.

7. Glevaskiy IV. Beet growing. Kyiv: Vyshcha shkola, 1991. 620 p.

8. State Statistics Service of Ukraine. URL: http://www.ukrstat.gov.ua.

9. Sabluk PT, Kodenska M.Yu., Vlasov VI. Sugar beet production in Ukraine: revival problems, development prospects: monograph. In: PT Sabluk, MYu Kodenska, eds. Kyiv: NSC IAE, 2007. 390 p.

10. Danylyshyn MS. Efficiency of the sugar beet industry of Ukraine. Ekonomika ta derzhava. 2016; 1: 108-111.

11. Royik MV, Kornyeyeva MO. Sugar beet hybrids of new generation and their role in the process intensification of the branch. Plant Varieties Studying and Protection. 2006; 3: 71-82. DOI: 10.21498/2518-1017.3.2006.67681.

12. Chekalin MM, Tyshchenko VM, Batashova ME. Breeding and genetics of some crops. Poltava, 2008. 368 p.

13. Peretyatko VG, Borshkivsky IM. Breeding for improved shape and size of root vegetables. Tsukrovi buryaky. 2002; 3: 16-21.

14. Royik MV, Zuyev MM, Borysyuk VO. On morphological characteristics of sugar beet plants and their impact on the performance and quality of mechanized harvesting. Zbirnyk naukovykh prats Instytutu tsukrovykh buryakiv UAAN. 2000; 2(2): 146-166.

15. Royik MV, Kornyeyeva MO. Beetroot shape as an important ecological and breeding feature of sugar beets. Increase in the efficiency of beet production and problems of ecology and waste. Pidvyshchennya efektyvnosti buryakovoho vyrobnytstva ta problemy ekolohiyi i vidkhodiv. Kyiv, 1994. P. 33-34.

16. Tsialtas JT, Maslaris N. Sugar beet root shape and its relation with yield and quality. Sugar Tech. 2010; 12(1): 47-52.

17. Khomenko OI, Pushanko MM. Relationships between geometric characteristics and associated soilness of sugar beetroots. Kharchova promyslovist. 2008; 6: 12-15

18. Royik MV, Gisbullin NG, Sinchenko VM, Prysiazhnyuk OI. Research methods in beet growing. In: Royik MV, Gizbullyn NG, editors. Kyiv : FOP Korzun DYu, 2014. 374 p.

19. Dospekhov BA. Methods of field experiments with the basic of statistical procesing of research resalts. Moscow: Agropromizdat, 1985. 351 p.

\section{ПРОДУКТИВНИЙ ПОТЕНЦАЛ ЦУКЕРОВИХ БУРЯКІВ $З$ ПОЛІПШЕНОЮ ФОРМОЮ КОРЕНЕПЛОДУ В СЕЛЕКЦІЇ НА ГЕТЕРОЗИС}

Парфенюк О.О., Труш С.Г.

Дослідна станція тютюнництва ННЦ «ІЗ НААН», Україна

Мета дослідження - підвищення продуктивності гібридів цукрових буряків із застосуванням комплексних підходів формування, ідентифікації та добору комбінаційноцінних батьківських компонентів. Оцінка продуктивного потенціалу експериментальних гібридів цукрових буряків, створених на основі батьківських компонентів з поліпшеною формою коренеплоду.

Матеріали і методика. Дослідження проводили на Дослідній станції тютюнництва ННЦ «IЗ НААН» у лабораторії селекції цукрових буряків у 2018-2020 рр. Вихідним матеріалом для польових дослідів були 66 експериментальних диплоїдних гібридів цукрових буряків, створених на основі батьківських компонентів різного генетичного походження.

Створення нового вихідного матеріалу цукрових буряків проведено із застосуванням класичних методів селекції за загальноприйнятими методиками. Експериментальні гібриди цукрових буряків на ЦЧС основі досліджено за комплексом цінних господарських ознак відповідно до методик сортовипробування, розроблених в Інституті біоенергетичних культур 
та цукрових буряків НААН.

Обговорення результатів. Аналіз продуктивності гібридів цукрових буряків, створених на основі батьківських компонентів з поліпшеною формою коренеплоду (овальноконічною) доводить, що за врожайністю коренеплодів, збору та виходу цукру вони істотно перевищують груповий стандарт. Цукристість цих коренеплодів була нижчою або на рівні групового стандарту. В цілому, гібриди характеризуються врожайною спрямованістю (Е) продуктивності. За врожайністю коренеплодів вони перевищували груповий стандарт на $16,5-23,4$ \%, збором цукру - на 15,1-21,1 \% та виходом цукру - на 15,0-22,0 \%.

За аналізом параметрів форми коренеплоду гібридів даного типу встановлено, що вони $\epsilon$ овально-конічними. Показники індексу форми коренеплоду в них варіювали в межах $1,11-$ 1,24. Коренеплоди цих гібридів заглиблюються в землю на 3/4 довжини, мають гладеньку поверхню та мілку кореневу боріздку (ортостиху).

В експериментальних гібридів цукрових буряків, сформованих на основі батьківських компонентів 3 поліпшеною формою коренеплоду (овально-конічною), збільшувалась довжина коренеплоду (L) на 9,5\%, його діаметр (D) - на 2,2\%, відстань від площини максимального діаметра коренеплоду до вершини голівки (B) на 61,5\% у порівнянні 3 груповим стандартом. Зі зміною параметрів форми коренеплоду збільшилася і його маса $(\mathrm{m})$ на 25,0 \%. Установлено, що зміна форми коренеплоду батьківських компонентів 3 конічної на овально-конічну призводить до підвищення продуктивності гібридів цукрових буряків на $15-21 \%$.

Висновки. Створено 14 високопродуктивних гібридів цукрових буряків на ЦЧС основі з поліпшеною формою коренеплоду, придатних для енерго- та екологозберігаючих технологій вирощування. Вони перевищують груповий стандарт за врожайністю коренеплодів на 16,5-23,4 \%, збором цукру на 15,1-21,1 \% та виходом цукру на 15,0-22,0 \%.

Ключові слова: иукрові буряки, вихідний матеріал, гібрид, гетерозис, форма коренеплоду, індекс форми, продуктивність.

\section{ПРОДУКТИВНЫЙ ПОТЕНЦИАЛ САХАРНОЙ СВЕКЛЫ С УЛУЧШЕННОЙ ФОРМОЙ КОРНЕПЛОДА В СЕЛЕКЦИИ НА ГЕТЕРОЗИС}

Парфенюк О.А., Труш С.Г.

Опытная станция табаководства ННЦ «Институт земледелия НААН», Украина

Цель исследований - повышение продуктивности гибридов сахарной свеклы с использованием комплексных подходов формирования, идентификации и отбора комбинационно-ценных родительских компонентов. Оценка продуктивного потенциала экспериментальных гибридов сахарной свеклы, созданных на основе родительских компонентов с улучшенной формой корнеплода.

Материалы и методика. Исследования проводили на Опытной станции табаководства ННЦ «ИЗ НААН» в лаборатории селекции сахарной свеклы в 2018-2020 гг. К полевым опытам привлечены 66 экспериментальных диплоидных гибридов сахарной свеклы, созданных на основе родительских компонентов разного генетического происхождения.

Создание нового исходного материала сахарной свеклы проведено с использованием классических методов селекции по общепринятым методикам. Экспериментальные гибриды сахарной свеклы на ЦМС основе изучены по комплексу ценных хозяйственных признаков соответственно методикам сортоиспытания, разработанных в Институте биоэнергетических культур и сахарной свеклы НААН.

Обсуждение результатов. Анализ продуктивности гибридов сахарной свеклы, созданных на основе родительских компонентов с улучшенной формой корнеплода (овально-коническая) показывает, что по урожайности корнеплодов, сбору и выходу сахара они существенно превышают групповой стандарт. Сахаристость их корнеплодов была ниже или на уровне группового стандарта. В целом, гибриды характеризуются урожайным 
направлением (Е) продуктивности. По урожайности корнеплодов они превышали групповой стандарт на 16,5-23,4 \%, сбору сахара на 15,1-21,1 \% и выходу сахара на 15,0-22,0 \%.

По анализу параметров формы корнеплода гибридов данного типа установлено, что они характеризуются овально-конической формой. Показатели индекса формы корнеплода в них варьировали в пределах 1,11-1,24. Корнеплоды этих гибридов заглублены в почву на 3/4 длины, имеют гладкую поверхность и мелкую корневую бороздку (ортостиху).

У экспериментальных гибридов сахарной свеклы, сформированных на базе родительских компонентов с улучшенной формой корнеплода (овально-коническая), увеличилась длина корнеплода (L) на 9,5 \%, диаметр корнеплода (D) - на 2,2 \%, расстояние от плоскости максимального диаметра корнеплода до вершины головки (B) на 61,5 \% по сравнению с групповым стандартом. С изменением параметров формы корнеплода увеличилась и его масса $(\mathrm{m})$ на $25,0 \%$. Установлено, что изменение формы корнеплода родительских компонентов с конической на овально-коническую приводит к повышению продуктивности гибридов сахарной свеклы на 15-21\%.

Выводы. Создано 14 высокопродуктивных гибридов сахарной свеклы на ЦМС основе с улучшенной формой корнеплода, пригодные для энерго- и экологощадящих технологий выращивания. Они превышают групповой стандарт по урожайности корнеплодов на 16,5$23,4 \%$, сбору сахара на 15,1-21,1 \% и выходу сахара на $15,0-22,0 \%$.

Ключевые слова: сахарная свекла, исходный материал, гибрид, гетерозис, форма корнеплода, индекс формы, продуктивность.

\section{PERFORMANCE POTENTIAL OF SUGAR BEETS WITH IMPROVED BEETROOT SHAPE IN BREEDING FOR HETEROSIS}

Parfeniuk O.A., Trush S.H.

Tobacco Experimental Station of the National Research Center "Institute of Agriculture of NAAS", Ukraine

Purpose. To increase the performance of sugar beet hybrids using integrated approaches to the creation, identification and selection of combination-valuable parents; to evaluate the performance potential of experimental hybrids derived from parents with improved beetroot shape.

Materials and methods. The study was conducted in the Laboratory of Sugar Beet Breeding of the Tobacco Experimental Station of the National Research Center "Institute of Agriculture of NAAS" in 2018-2020. Sixty-six experimental diploid sugar beet hybrids originating from parents of different genetic origin were tested in the field.

New starting material of sugar beets was created using classical breeding methods and traditional techniques. The experimental CMS-based sugar beet hybrids were evaluated for several economically valuable traits in accordance with the variety trial method developed by scientists of the Institute of Bioenergy Crops and Sugar Beets NAAS.

Results and discussion. Analysis of the performance of the sugar beet hybrids originating from the parents with improved beetroot shape (oval-conical) indicates that the beetroot yields, sugar collection and output were significantly higher than those in the group check accession. The sugar content in their beetroots was lower than or similar to that in the group check accession. In general, the yielding type (E) of performance is intrinsic to the hybrids. As to beetroot yield, they exceeded the group check accession by $16.5-23.4 \%$; the sugar collection was higher by $15.1-$ $21.1 \%$; and the sugar output - by $15.0-22.0 \%$.

Analysis of the beetroot in these hybrids shows that they had oval-conical beetroots. Their shape index varied 1.11 to 1.24 . The beetroots of these hybrids were embedded into the soil by $3 / 4$ of their length, had a smooth surface and shallow grooves (orthostichy).

In the experimental sugar beet hybrids derived from the parents with improved beetroot shape (oval-conical), beetroots were longer (L) by $9.5 \%$, larger in the diameter (D) by $2.2 \%$, and the 
distance from the maximum diameter plane to the beetroot top (B) increased by $61.5 \%$ compared to the group check accession. In parallel with changes of the beetroot shape, the beetroot weight $(\mathrm{m})$ increased by $25.0 \%$. The transition from conical beetroots to oval-conical ones was proven to increase the performance of the sugar beet hybrids by $15-21 \%$.

Conclusions. We have created 14 high-yielding sugar beet CMS-based hybrids with improved beetroot shape, suitable for energy and environmentally friendly cultivation technologies. Their beetroot yields, sugar collection and sugar output exceed those in the group check accession by $16.5-23.4 \%, 15.1-21.1 \%$ and $15.0-22.0 \%$, respectively.

Key words: sugar beets, starting material, hybrid, heterosis, beetroot shape, shape index, performance. 